\title{
Political Opinion ANALYSis in Social NETWORKS: CASE OF TWITTER AND FACEBOOK
}

\author{
Telesphore TIENDREBEOGO and Yassia ZAGRE \\ University of Nazi Boni, Bobo Dioulasso, Burkina Faso
}

\begin{abstract}
The $21^{\text {st }}$ century has been characterized by an increased attention to social networks. Nowadays, going 24 hours without getting in touch with them in some way has become difficult. Facebook and Twitter, these social platforms are now part of everyday life. Thus, these social networks have become important sources to be aware of frequently discussed topics or public opinions on a current issue. A lot of people write messages about current events, give their opinion on any topic and discuss social issues more and more.

The emergence and enormous popularity of these social networks have led to the emergence of several types of analysis to take advantage of them. One of them is the analysis of opinions in texts. It aims at automatically classifying opinions in order to position them on a sentiment scale, thus allowing to characterize a set of opinions without having to rely on a human to read them. Currently, opinion analysis offers us a lot of information related to public opinion, either in the commercial world or in the political world. Many studies have shown that machine learning techniques, such as the support vector machine $(S V M)$ and the naive Bayes classifier (NB), perform well in this type of classification.
\end{abstract}

In our study, we first propose an approach for tracking and analyzing political opinions in social networks. Then, we propose a trained and evaluated machine learning model for political opinion classification. And finally, the study aims at setting up a web interface to collect and analyze in real time political opinions from social networks

\section{KEYWORDS}

Opinion analysis, opinion mining, Naive Bayes, Support Vector Machine, text classification, social networks, machine learning, polarity

\section{INTRODUCTION}

Lately, social networks have become deeply rooted in the lives of their users. According to Hootsuite[1] in January 2020 there were 3.8 billion active users in the world of social networks. On the same date, Burkina Faso had 1.6 million active users of social media with a growth of $37 \%$ from 2019 to 2020 . As a result, users are not only expressing their opinions on any topic, but also increasingly discussing social issues and political events These exchanges create a large volume of potentially exploitable information.

The development of social networks, which can be considered as a democratization of computing, generates a rich mine of exploitable data called "Big Data". This data is critical to decision making for many individuals and organizations[2]. For this purpose, new analysis technologies, alternatives to polls, have indeed appeared in recent years within the media and political fields. As a result, researchers have since tried to conceptualize methods to analyze this "Big Data" in a methodical, structured and efficient way[3]. Thus, among many methods, opinion analysis has been developed to specifically analyze the polarity (i.e.: positive or negative) of texts 
International Journal of Web \& Semantic Technology (IJWesT) Vol.12, No.4, October 2021

posted online on various levels. The objective of this type of analysis is generally to classify texts or parts of texts according to the polarity of their author with respect to a given topic.

Our objective in this paper is to propose on the one hand an approach to monitor and analyze political data generated in social networks. On the other hand, we propose a machine learning model to analyze political opinions from social networks.

Our paper is structured as follows: we first present related work on opinion analysis. We then provide an overview of techniques and applications. Finally, we present and explain the implementation of a machine learning model for the analysis of our data. We also offer a web interface to collect and analyze political opinions from social networks in real time.

\section{RELATED WORKS}

\subsection{Social Networks}

Social networks such as Twitter and Facebook have been the subject of a lot of research. Several main factors explain this interest. The first is that these networks are almost entirely composed of user-generated content. This would mean that every message posted on these sites has an opinion or subjectivity of some kind. Moreover, the user is free to post whatever he or she wants, which generates very diverse themes and topics, most of which are related to current events. In [2], the authors point out that this portrait of opinion at a specific moment in time can be used to analyze the evolution of opinion on certain subjects, provided that a periodic analysis of the latter is performed. Secondly, the majority of social networks make their APIs available which facilitates data collection as mentioned by Fang and Zhan (2015) [4]. Finally, the number of active people online, who generate content, is massive [5]. Such social networks are therefore real data mines that allow researchers, individuals as well as large companies to collect data among a wide range of topics.

\subsection{Social Networks and Political Opinions}

The potentials of social media seem to be the most promising in the political context as they can be a catalyst for more participation and democracy [6]. In [7], Stefan Stieglitz defines public participation as the process by which the concerns, needs, and values of the public are incorporated into the decision-making of governments and corporations. In a similar vein, Karpf[8] introduces the notion of "Political 2.0" which can be understood as exploiting the reduced transaction costs of the Internet and its abundance of information, in order to create more participatory and interactive political institutions. There is a large body of research regarding the role of social networks in political thinking. The US presidential campaign revealed that social networks have become increasingly essential to political communication and persuasion. Recent studies have shown an emerging need for political institutions as well as government departments to leverage the resources of social networks to improve services and communication with citizens and voters [8]. Similarly, Williams and Gulati [9] assert that it has become increasingly important to stay abreast of ongoing discussions and manage one's own reputation in virtual communities, especially with respect to emerging topics that may lead to scandal or crisis for a specific politician or party. In a survey of members of the German "Bundestag", Stieglitz and Stefan [8] found that most members of parliament would like to benefit from concepts and instruments to identify sensitive political topics early on. However, this task requires a lot of effort, appropriate tools and, above all, a systematic approach. 


\subsection{Opinion Analysis}

Interest in the subject of sentiment analysis seems to be increasing year after year since 2002. At least that's what we could tell by looking at the evolution of the search for the phrase "sentiment analysis" in the google search engine. See Figure 1.

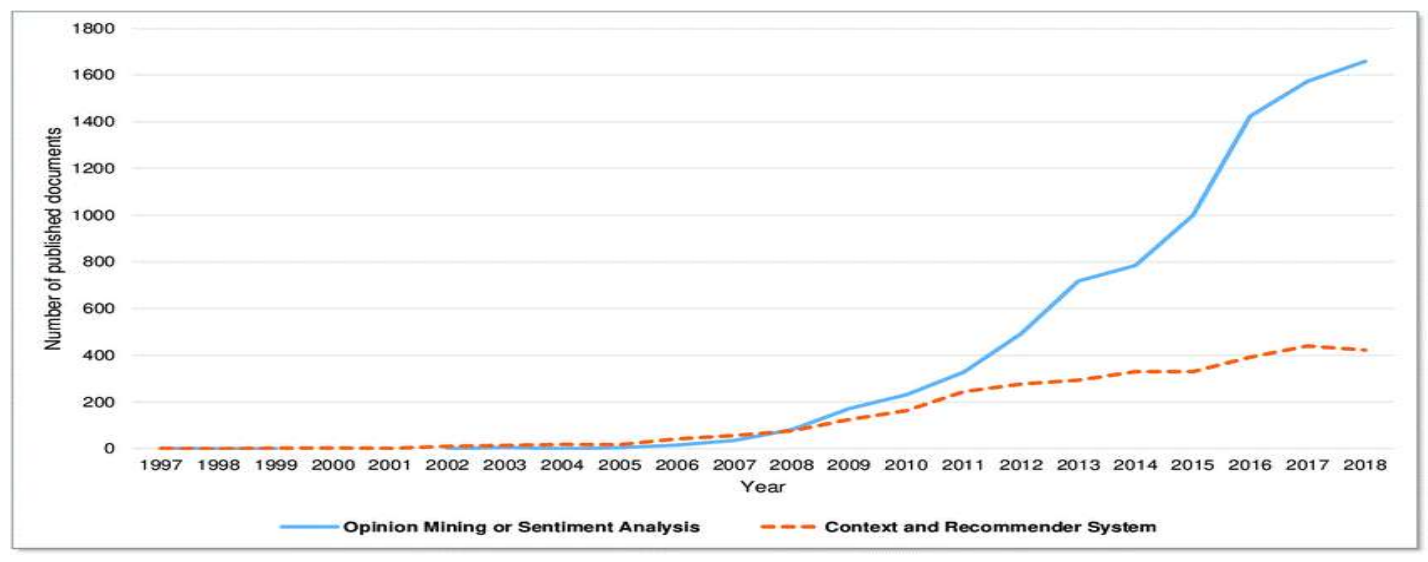

Figure 1: Evolution of searches made with the expression sentiment analysis in the Google search engine since 2002in the world

It seems that the attraction for this topic is also shared by the research world. Indeed, more than 7000 articles have been published on this subject until 2019[10]. The analysis of the opinion thus occupies a place of a certain importance from the interest that is granted to it and by all the literary activity that is dedicated to it. In the literature, sentiment analysis, opinion mining, opinion extraction, sentiment mining, subjectivity analysis, emotion analysis, review mining, are terms used to designate technologies for the automatic analysis of written or spoken discourse in order to extract subjective information such as judgments, evaluations or emotions.

According to Cambria[11], opinion analysis has been one of the most active research areas in natural language processing since the early 2000 . The purpose of opinion analysis is to design automatic tools to extract information from a natural language text, such as opinions and feelings, in order to generate knowledge that can be used by a decision support system or by a decision maker. Opinion analysis is the computer processing of a text that is explored in order to identify the general sentiment or opinion expressed. We often speak of classifying the polarity (positive, negative or neutral) of a text. The exploration is done on an entity and these entity can represent individuals, products, events or various subjects that are likely to be covered by comments, criticisms or appreciations.

In order to identify the polarity of a text, opinion analysis relies on several features which are among others: the frequency of a term, the importance of a term in the text, negations in sentences [12]. In other words, opinion analysis concerns the processing of an opinion text to extract and categorize the opinions of a certain document. The polarity of the sentiments usually expressed in terms of positive or negative opinion (binary classification).However, there can be a multiple classification, where the feeling can have a neutral label or even a different label like very positive, positive, neutral, negative, very negative, the labels can also be associated with emotions such as sadness, anger, happiness, etc. 


\subsubsection{Opinion Analysis Levels}

The research on opinion analysis was mainly conducted at three different levels of analysis [2]: Message level or Document level, Sentence level and aspect level.

\subsubsection{Opinion Analysis and Applications}

According to Pang and Lee [2], it was in 2001 that the field of automatic opinion analysis began to attract attention. The numerous possibilities brought by this method and the doors it could open eventually aroused the interest of many political, sociological, governmental, financial, advertising and commercial fields.

- Politics: Nowadays, political actors follow the trend of opinion analysis, because before declaring a new law, politicians try to collect the opinion of social network users about this law. Through opinion analysis, policy makers can collect citizens' opinions on certain policies, in order to benefit from this information to improve or create a new policy that suits with citizens. It is highly strategic to also know the opinion of Internet users on a politician during a presidential election [13][14]. It should be noted that people's opinion and experience is a very useful element in the decision-making process.

- Economy: Thanks to the analysis of opinion, the companies can know the opinion of the customer son their products or their services. In a perspective of improvement of their products and increase of their sales and incomes. Marketing has quickly understood the interest of opinion analysis. An example of commercial interest found in Pang and Lee's study [2] is that of a marketer wanting to know why his new product was not selling well. $\mathrm{He}$ was therefore interested in the opinions of his consumers regarding his product. The result was useful for him because by knowing their opinions, he was able to orient his offer according to the requirements of the market.

- Other areas of application: Recently, opinion analysis is also being applied in totally different fields, namely on-profit fields. The two most sought-after domain sat the moment are the detection of cyber stalking as well as the detection of suicidal tendencies of people active on the web[15][16][17][18].

\subsubsection{Opinion Analysis Approach}

In the literature review, there are generally three approaches to opinion analysis:

- Lexicon based approach;

- Machine Learning based approach;

- Hybrid approach

Lexical approach: According to Annett and Kondrak, Sasikala and Mary, the lexical approach consists of identifying words present in a dictionary of pre-labeled words (each assigned a value on a scale composed of several sentiment intensities) and tallying the sentiment numbers to arrive at a total polar score for each text [19][20]. This approach therefore requires the availability of a dictionary that is adapted to the language used in the texts understudy. Moreover, according to Muhammad et al, for the dictionary to be effective, it must be adapted to the domain under study [21].

Machine learning approach: A major shortcoming of this lexicon-based approach is its inability to identify opinion words with domain- and context-specific orientations. As, a sub-field of artificial intelligence $(\mathrm{AI})$, machine learning based approach refers to the techniques and actions 
International Journal of Web \& Semantic Technology (IJWesT) Vol.12, No.4, October 2021

that researchers have to develop an automatic system that, by analyzing data, learns by itself. These learning algorithms allow systems to train themselves with the data provided in order to develop and improve. From a set (or model) of data called "dataset", the algorithms are trained and tested. the algorithms are trained and can, from these models, make predictions about other data they may encounter in the future. The machine learning approach is subdivided into supervised, unsupervised and semi supervised learning methods.

Hybrid Approach: This approach combines the lexicon based approach and the machine learningbased approach and tries to correct the drawback of the lexicon-based approach of low recall and the machine learning-based approach of manual annotation.

Classification algorithms: There are different classifiers in machine learning methods that are used for opinion analysis. The Support Vector Machine method was introduced by Joachims [22][23], then used by Drucker [24], Taira and Haruno [25], and Yang and Liu[26]. The Naïve Bayes classifier is a supervised probabilistic learning technique that is regularly used for classification purposes. It is based on Bayes' theorem where the conditional probability of an event $\mathrm{X}$ occurring, for an index Y, is determined by Bayes' rule [2] (See equation 1).

$$
\mathrm{P}(\mathrm{X} / \mathrm{Y})=\mathrm{P}(\mathrm{X}) \mathrm{P}(\mathrm{Y} / \mathrm{X}) / \mathrm{P}(\mathrm{Y})
$$

The k-Nearest Neighbors KNN method is a classification method in which the model stores observations from the training set for classification of the test set data. A text classifier based on the decision tree method is a tree of internal nodes that are labeled with terms, the branches coming out of the nodes are tests on the terms, and the leaves are labeled with categories [27].Neural networks constitute a network of units, where input units correspond to terms, output units to categories of interest, and weights of edges connecting units to dependency relationships. To rank a test document d_i, its weights W_ki are loaded into the input units; activation of these units propagates through the network, and the value of the output unit(s) determines the ranking decision [4].

\subsubsection{Opinion Analysis Problems}

In the literature, opinion analysis is a very complex task with many unsolved problems[2]. The following are some of the challenges in dealing with this area. The handling of comparisons and the handling of negation, the detection of false opinions [2], the problem of language, Aboutness, the detection of sarcasm[28].In this section, we have first presented what opinion analysis is, with its three different levels of analysis and its applications. Then, we presented the approaches that exist in the literature with the different algorithms.

And finally, we evoked according to the literature the problems which existed in this type of task. Studies have shown that social networks such as Facebook and Twitter are a reliable indicator of the general opinion of users on entities, events or institutions, etc.

\section{Methodology}

In this section, we propose an approach to tracking and analyzing political opinions from Twitter and Facebook. While data tracking and monitoring concerns different approaches to how and what kind of politically real data generated by users of different social network platforms can be tracked and collected, the section on data analysis discusses different methods of analysis for different purposes and approaches. 
International Journal of Web \& Semantic Technology (IJWesT) Vol.12, No.4, October 2021

\subsection{Data Tracking Approach}

Tracking sources: The first step in data collection is to determine the sources of the data. For this reason, we decided to develop our approach by focusing on Twitter and Facebook as the primary sources of social media detain the case of Twitter, the data to be monitored and exploited is in the form of public "tweets" that are easily accessible. For Facebook, the most important type of data is the content of the "wall", including "status updates" and related comments. It is important to note that data on Facebook is, unlike Twitter, semi-public in nature,i.e., data can only be collected from public profiles (or more concretely, groups or "pages", since most individual profiles are not public). Specifically, in a political context, a list of politically relevant Facebook groups and pages should be predefined as data sources. Tracking methods: Both Twitter and Facebook offer application programming interfaces (APIs) for tracking data. The most frequently used APIs for Twitter are the Search API and the Streaming API, while Facebook's Graph API allows programmers to conveniently track wall posts (i.e. status updates and comments).

Tracking Approaches: We have identified four approaches to tracking political data, which depend on the specific intentions of the tracker: (1) self-reputation,(2) keyword/topic-based, (3) actor-based, and (4) random/exploratory.

- self-reputation Approach: The first approach is applicable when, for example, politicians or political parties want to know explicitly how people talk about them in social networks. In this case, politicians or parties can have all tweets that contain their name collected, either as a simple keyword or as a hashtag. If they have their own presence on Facebook in terms of a page or group, they should follow all posts and corresponding comments published by users or members of their own page or group.

- Keyword/Topic-based Approach: Politicians are often very interested in the reactions or opinions of Internet users on certain political topics. In this case, the second tracking method seems appropriate, as it tracks Tweets as well as Facebook posts that contain keywords related to the topics of interest. To achieve a high level of data completeness, the relevant keywords of the topic of interest must be carefully selected. The more important the topic to be analyzed, the higher the number of keywords to be taken into account.

- Actor-based Approach: In political communication, a number of actors can generally be considered more influential or popular than most other users. These actors are said to have the power to influence opinion-forming processes (online)[2]. Therefore, politicians or political parties are also interested in tracking these important users in terms of generated content. For this, an actor-based tracking approach could be used to track tweets, wall posts as well as corresponding comments specifically provided by these influential users who should also be identified in advance.

- Random/Exploratory Approach: Unlike the three tracking approaches that are more targeted in nature, the fourth approach supports content mining. The principle of this tracking approach is to randomly determine one or more data sets (tweets or Facebook posts) for different time periods for analysis. Based on this random data, a content analysis can be used to identify key political topics and detect the opinions or feelings of users associated with these topics.

\subsection{Opinion Analysis Approach}

A major drawback of the lexicon-based approach is its inability to identify opinion words with domain- and context-specific orientations. Based on the difficulties of the lexicon-based method reported in the literature review, it is appropriate to use a machine learning technique. For this purpose, we have chosen machine learning algorithms according to their popularity in the 
literature and according to their predictive potential in the context of opinion analysis. We chose four algorithms, including support vector machines (SVM), decision tree (DT), random forest $(\mathrm{RF})$ and Naïve Bayes (NB). Random Forest (RF) and Naïve Bayes (NB). Finally, we compared the classification results of the four algorithms in order to select the one that gives the best result in the classification of political opinions.

\subsection{Data Representation}

The data representation methodology that we adopt takes into account the information that was synthesized in our literature review. Indeed, we have chosen to prepare the data according to the types of representations and transformations that we thought were the best given the characteristics of our data, namely Term Frequency Inverse Document Frequency (TF-IDF). Considering the terms $\mathrm{j}$ in a document $\mathrm{i}$ being part of a certain corpus of documents, here is how we could represent the TF-IDF under an algebraic form[4] (see equation 2).

$$
\boldsymbol{V}_{i j}=\boldsymbol{T} \boldsymbol{F}_{\boldsymbol{i j}} . \boldsymbol{I D F _ { j }} \text { such as } \boldsymbol{T} \boldsymbol{F}_{i j}=\frac{f_{i j}}{n_{i}} \text { and } I D F_{j}=\log \left(\frac{m}{\sum_{i=1}^{m} b_{i j}}\right)
$$

where:

$\boldsymbol{V}_{\boldsymbol{i} j}=$ the value of the term $\mathrm{j}$ in the document $\mathrm{i}$.

$\boldsymbol{f}_{\boldsymbol{i j}}=$ number of times the term $\mathrm{j}$ in the document $\mathrm{i}$.

$\boldsymbol{n}_{\boldsymbol{i}}=$ total number of terms in the document $\mathrm{i}$.

$\boldsymbol{b}_{\boldsymbol{i} j}=1$ if the term $\mathrm{j}$ is present in the document $\mathrm{i}$ and 0 else.

$\boldsymbol{m}=$ if the term $\mathrm{j}$ is present in the corpus.

\subsection{Data Pre-Processing}

The characteristics of tweets generally boil down to their limited length and the use of informal language. Thus, Twitter users use abbreviations, emoticons, and slang to express their opinions and feelings. Therefore, a pre-processing step is essential. In the following we present the preprocessing procedure followed in our work, whose goal of this step is to clean up the tweets and make them as close as possible to a formal language. First we started by eliminating URLs and words with hashtag, non-alphabetic characters, repeated terms and punctuations.

\subsection{System Architecture}

Our study focuses on the task of opinion analysis on social network data in particular Twitter and Facebook. To achieve this goal and to get the best possible performance, we propose the following architecture. Figure 2 below illustrates the general process of the system which includes several steps including data collection, pre-processing, annotation, sentiment analysis and visualization. 


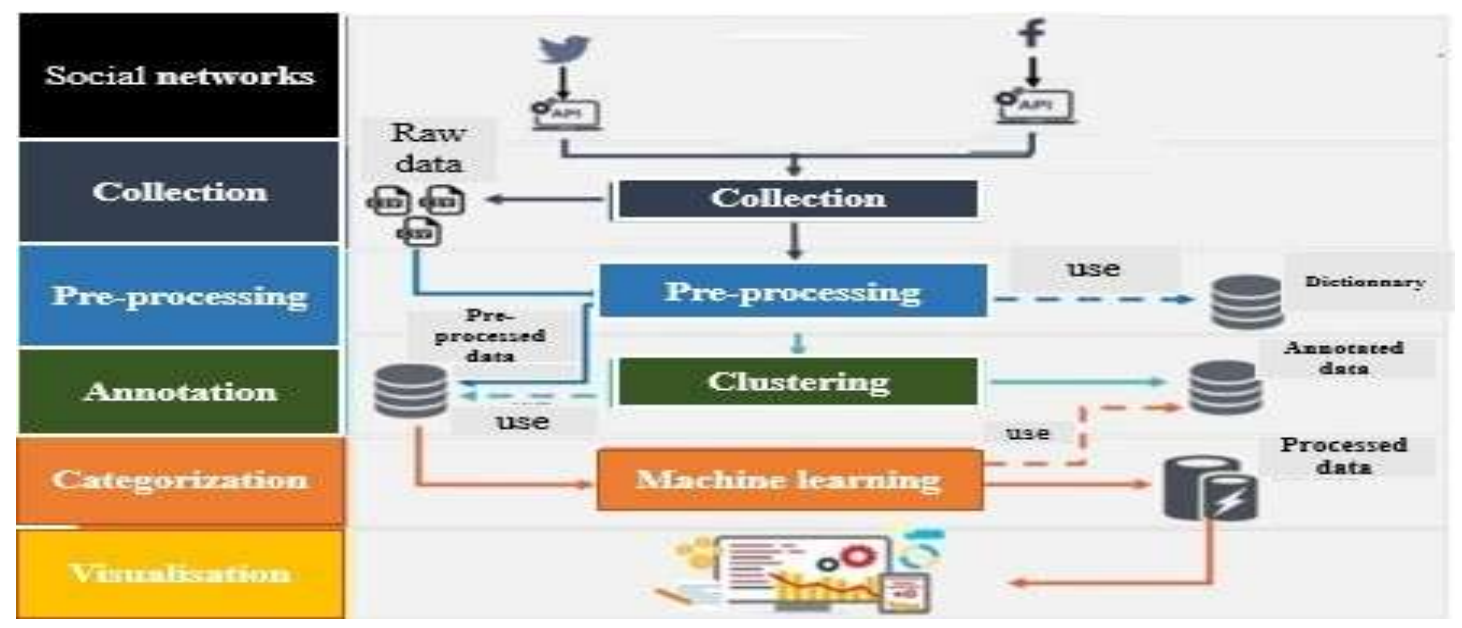

Figure 2: System architecture

The collection phase scans Twitter and Facebook to extract tweets and comments thanks to the API of the social network. The result of the collection goes through a step of pre-processing which allows to remove the duplicates and the spams (same author, same content, same post). Once the data has been pre-processed, comes the annotation phase, which allows to create a training dataset for the opinion analysis system. The text stream from the comments is then sent to categorization to determine the sentiment class, which in turn uses the data from the annotation layer for learning. The visualization phase draws from the latter to expose the results to the user according to the desired parameters. The final application will take the form of a Dashboard that present she aggregated statistics of the analysis. In this section, we have proposed an approach to tracking and analyzing political opinions from social media. Specifically, we have described various data tracking approaches and corresponding analysis methods that could help to better understand political discussions in socialmedia. From a practical perspective, this approach should serve as a guideline for developing tools to collect, store, analyze, and summarize usergenerated social media content that is politically relevant to political institutions. From a research perspective, the framework provides a comprehensive overview of different specific methodological approaches that can be used to analyze political opinions from social networks.

\section{BACKGROUND}

These first years of the 21 st century seem to be marked by multiple changes in the production and exchange of information. Thus, since the advent of Web 2.0 and especially the massive use of social networks, the number of users has considerably increased and their role has evolved into that of information provider. As a result, an increasing number of users send hundreds of millions of messages every day to specially created websites, such as Facebook and Twitter. Users write about their vision of society, increasingly discuss social issues and political events, share opinions and divulge ideas [4].

As a result, social networks have become an essential research material for researchers in computer science and the humanities. To this end, new analysis technologies, alternatives to polls and surveys which are reputed to be long and costly, have indeed appeared in recent years within the media and political fields. Thus, among many methods, opinion analysis has been developed to specifically analyze the polarity of texts. Hence the growing development of techniques to capture these evaluations of Internet users, ranging from the simple counting of positive or negative comments to the more detailed analysis of the contents of these comments. It is in this 
International Journal of Web \& Semantic Technology (IJWesT) Vol.12, No.4, October 2021

context that this paper is written. The objective of this paper is to propose an approach to monitor and analyze political data generated in social networks. On the other hand, we propose a machine learning model to analyze political opinions from social networks.

\section{RESUlTS AND DiscuSSION}

In this section, we first present the data that was used to train our model. The input data we used are tweets, extracted from a dataset to train and test our model. We also test the proposed model in real time on tweets extracted from Twitter itself. These tweets represent the statuses posted on this social network and related to the political field.

\subsection{Dataset}

To train our algorithms, it is important to have well annotated data. To do so, we thought it wiser to take texts already marked by their authors rather than marking +9 them ourselves. For the sake of rigor, we also considered the size of the sample as an essential factor to improve the value of the results. Our choice was therefore theSentiment140 dataset which is part of the Sentiment140[29] project at Stanford University. Indeed, this database contains thousands of tweets with their respective ratings, and therefore has the necessary assets for the application of our classification. It is a large dataset, more than one million tweets with two classes of negative and positive tweets, with 800,000 for each. The authors represented the positive class with the number 4 and the other class with the number 0 .

\subsection{Presentation of Results}

Recall that the objective of our study is to implement a system to classify tweets or comments on political topics. Such a system consists of two phases in the implementation. The first step is a machine learning model trained with algorithms and among them the one that performs best. The second phase is the implementation of a web application using a machine learning model and allowing to visualize the trends in real time. We will present here the results of the machine learning models.

For the validation of the performances of our model, we use the $80 \% 20 \%$ method, as $80 \%$ used in the learning phase, and $20 \%$ for the testing phase. In agreement with [30]. Table I presents the confusion matrix that applies to our binary classification context. The performance measures used are precision, recall and F1-measure, whose bases of calculation are relative to the confusion matrix.

Table 1 : Confusion matrix in a polar classification contexte.

\begin{tabular}{|l|l|l|}
\hline & Positive & Negative \\
\hline Positive & True Positive (TP) & False Positive (FP) \\
\hline Negative & False Negative (FN) & True Negative (TN) \\
\hline
\end{tabular}

The measurements are presented in the following formulas:

$$
\text { Accuracy }=\frac{T P}{T P+F P}(3)
$$

This measure represents the proportion of correct predictions to the total number of predictions. predictions. 
International Journal of Web \& Semantic Technology (IJWesT) Vol.12, No.4, October 2021

$$
\text { Recall }=\frac{\mathrm{TP}}{(\mathrm{TP}+\mathrm{F} \mathrm{N})}(4)
$$

This metric corresponds, for a binary classification, to the number of positive predictions of the model out of the total of predictions that should be positive. Otherwise, it is the proportion of well-ranked items to the number of items in the class being predicted.

To combine all this information, we often introduce a metric called the F1-Score. It is calculated in this way.

$$
\mathbf{F 1}-\text { score }=\frac{1}{\frac{1}{1}\left(\frac{1}{\text { Recall }}+\frac{1}{\text { Accuracy }}\right)}
$$

$=2 *$ Accuracy $*$ Recall(Accuracy + Recall(4)

Represents the trade-off measure between precision and recall. In the table II, we summarize the result obtained for each model.

Table 2 : The validation results of dataset.

\begin{tabular}{|l|l|l|l|l|}
\hline Algorithms & NB & SVM & RF & DT \\
\hline Accuracy & $83.26 \%$ & $82.75 \%$ & $69.78 \%$ & $77.08 \%$ \\
\hline Recall & 0.7 & 0.8 & 0.6 & 0.7 \\
\hline F1-score & 0.7 & 0.8 & 0.6 & 0.7 \\
\hline CPU time & $1 \mathrm{mn} 50 \mathrm{~s}$ & $4 \mathrm{~h} \mathrm{54mn} \mathrm{30s}$ & $8 \mathrm{mn} 54 \mathrm{~s}$ & $2 \mathrm{~h} \mathrm{56mn} \mathrm{55s}$ \\
\hline
\end{tabular}

Our dataset consisted of nearly 1.6 million tweets evenly distributed in the 2 categories. In order to select the best algorithm, we used several different algorithms. Thus, after running several tests with different models and configurations, we selected Naïve Bayes as the best performing classifier for this particular problem. To evaluate the results, we used the 5-way cross-validation method and our best performing classifier reaches an accuracy of $83.26 \%$.

\section{Conclusions}

The arrival of the digital age, coupled with the rapid expansion of the web and its democratization, has profoundly changed the way society works. Now, the Internet is full of usergenerated content. This content, called "Big Data", has become an invaluable mine of data that can be exploited in many financial, political and no lucrative fields. At the same time, a number of methods to successfully analyze this data have emerged. Among these methods, opinion analysis has been developed to specifically analyze the polarity of texts posted online on various levels. These methods have become even more relevant due to the rise in popularity of social networks. The leaders in this field, Facebook and Twitter, have billions of users who are constantly generating new content while expressing their opinions.

In this paper, we focused on the analysis of political opinion in social networks. To achieve our goal, we proposed an approach for tracking and analyzing political opinion from social networks. Specifically, we have described various approaches to data tracking and the corresponding analysis methods On the other hand, we trained a machine learning model for political opinion analysis from social networks. To do so, we used four machine learning algorithms (SVM, DT, 
International Journal of Web \& Semantic Technology (IJWesT) Vol.12, No.4, October 2021

RF, NB) that we trained with the sentment140 [36] dataset. At the end of the training, we chose NB which is the best performing classifier in terms of accuracy. It reaches an accuracy of $83.26 \%$. Ultimately, the results obtained demonstrate that social networks, and Twitter and Facebook in particular, are a source of data for obtaining key indicators for better decision-making.

However, we could agree with some authors[3][4][12]that the opinions coming from social networks would not constitute a fairly representative sample of the distribution of the whole population, especially in our countries where the digital penetration rate remains very low, i.e. 22\% penetration rate in Burkina Faso with 1.6 million active users of social networks in January 2020 [31]. This could also be a significant bias. Nevertheless, we are witnessing a very rapid evolution of the penetration rate. This is the case in Burkina Faso, which has seen a strong 35\% grow thin active social media users from one year to the next [31].

As perspectives to our work, we foresee:

- test our model on other data sets;

- develop the model to manage the problem of sarcasm;

Finally, we believe that it is necessary to define a framework that will allow an efficient analysis of the data from social networks in order to derive several useful information for decision making.

\section{REFERENCES}

[1] Hootsuite, «Digital marketing and management,» janvier 2020. [En ligne]. Available: https://www.hootsuite.com/.

[2] L. L. a. B. Pang, «Opinion Mining and Sentiment,» in Foundation and Trends in information Retrieval, p. 63, 2008.

[3] D. B. a. A. Lohard, «Opinion mining and Sentiment analysis,» HEC MONTREAL, 2015.

[4] J. Labrecque, "Analyse des sentiments dans les textes à l'aide d'algorithmes d'apprentissage automatique,» HEC MONTREAL, 2015.

[5] F. X. a. Z. Justin, «Sentiment analysis using product review data,» Journal Of Big Data, 2015.

[6] A. M. K. a. M. Haenlein, «Users of the world nite! The challenges and opportunities of social media,» Business Horizons, 2009.

[7] A. M. K. a. M. Haenlein, «Users of the world unite! The challenges and opportunities of social media,» Business Horizons, 2009.

[8] D. A. Karpf, «Recherche sur la blogosphère : une approche à méthodes mixtes pour des systèmes en évolution rapide,» IEEE Intell Syst 24, 2009.

[9] C. B. a. G. J. Gulati, «Facebook grows up: An empirical assessment of its role in the 2008 congressional elections,» In Annual Meeting of the Midwest Political Science Association, 2008.

[10] F. Ronen, «Techniques and applications for sentiment analysis,» Commun.ACM, 2018.

[11] C. Erik, «Affective computing and sentiment analysis,» IEEE Intell, 2016.

[12] L. Bing, «Opinions, Sentiment, and Emotion in Text,» Cambridge University University Press, 2015.

[13] B. F. a. Sabatier, «Expérimentation de fonctions pour la détection d'opinions dans les Blogs,» Institut de Recherche en Informatique de Toulouse, 2010.

[14] E. C. a. D. O. a. D. Rajagopal, «SenticNet 3:a common and common-sense knowledge base for cognition driven sentiment analysis,» In Twenty-eighth AAAI conference on, 2014.

[15] V. H. a. C. L. a. E. B. V. a. J. M. a. B. D. a. D. G. P. a. V. Hoste, «Detection and finegrained classification of cyberbullying events,» In International In International, 2015.

[16] B. D. a. V. Hoste, «Online suicide prevention through optimised text classification,» Information Sciences, 439-440, 61-78., 2018.

[17] J. M. X. a. X. Z. a. A. Bellmore, «Fast learning for sentiment analysis on bullying,» In Proceedings of the First International Workshop on Issues of Sentiment Discovery and Opinion, 2012. 
International Journal of Web \& Semantic Technology (IJWesT) Vol.12, No.4, October 2021

[18] J. M. Gómez, «Language technologies for suicide prevention n social media,» In Proceedings of the First International Workshop on Issues of Sentiment Discovery and Opinion, 2014.

[19] M. A. a. G. Kondrak, «A comparison of sentiment analysis techniques : polarizing movie blogs,» In Proceeding of the Canadian Society for computational studies of intelligence, 21st conference on Advances in artificial intelligence., 2008.

[20] S. P. a. L. Mary, «Comparative Study of Sentiment Analysis Techniques in Web,» Immaculate Sheela. International Journal of Scientific Engineering Research, 2017.

[21] A. M. a. N. W. a. R. L. a. R. Glassey, «Domain-based lexicon enhancement for sentiment analysis,» 2017.

[22] J. Torsten, «Text categorization with support vector machines : learning with many relevant features,» 10th Euro pean Conference on Machine Learning, ECML-98, 1998.

[23] J. Torsten, «Transductive inference for text classification using support vector machines,,» 16th International Conference on Machine Learning, ICML-99, 1999.

[24] H. D. e. V. V. e. D. Wu, «Automatic text categorization and its applications to text retrieval,» IEEE Trans. Neural Netw, 1999.

[25] H. T. e. M. Haruno, «Feature selection in SVM text categorization,» 16th Conference of the American Association for Artificial Intelligence, AAAI-99, 1999.

[26] Y. Y. a. X. Liu, «A re-examination of text categorization methods,» 22nd ACM International Conference on Research and Development in Information Retrieval, 1999.

[27] T. Mitchel, «Machine Learning,» McGraw Hill, 1996.

[28] M. Abdelkader, «L'analyse du sentiment utilisant le deep Learning,» Université Dr. TAHAR MOULAY SAIDA, 2019.

[29] S. university, «trainingandtestdata,» 2012.

[30] M. S. e. N. J. e. S. Szpakowic, «Beyond accuracy, f-score and roc : A family of discriminant measures for performance evaluation,» In Australian Conference on Artificial Intelligence,volume 4304 de Lecture Notes in Computer Science, 2006.

[31] lekiosquedigitalduburkina.com, «lekiosquedigitalduburkina,» Business Horizons, 2018. [En ligne]. [Accès le janvier 2020].

[32] V. Antoine, «L'analyse des sentiments dédiée aux weets politiques : une approche par apprentissage automatique supervisé,» Aantal woorden:19.168, p. 19, 2017-2018.

\section{AUTHOR}

Yassia ZAGRE, Master degree in information systems and decision support system in nazi BONI university Burkina Faso. My interest research topic is image watermarking for decision support and multimedia system.

Telesphore TIENDREBEOGO, $\mathrm{PhD}$ and overlay network and assistant professor at Nazi Boni University. I have a master's degree in multimedia and real time system. My current research is on big data and image watermarking 